\title{
Preliminary numerical simulation investigation of the cone effect in an adaptive optics system using a laser guide star
}

\author{
Hai-Xing Yan*, Shu-Shan Li and She Chen \\ (Institute of Mechanics, Chinese Academy of Sciences, Beijing 100080, China)
}

\begin{abstract}
So-called cone effect or focus anisoplanatism is produced by the limited distance of a laser guide star (LGS) which is created within the Earth atmosphere and consequently located at a finite distance from the observer. We believe this is the first time to investigate the cone effect of the LGS by means of a pure numerical simulation. In this paper, the cone effect of the LGS for different vertical profiles of the refractive index structure constant $C_{n}^{2}$ is numerically investigated by using a revised computer program of atmospheric propagation of optical wave and an adaptive optics (AO) system including dynamic control process. It is surprisingly found that the effect of altitudes of the LGS on the AO phase compensation effectiveness by using the commonly-available vertical profiles of $C_{n}^{2}$ and the lateral wind speed in the atmosphere is relatively weak, and the cone effect for some $C_{n}^{2}$ profiles is even negligible. It is found that the cone effect seems not have obvious relationship with the turbulence strength, however, it depends on the vertical distribution profile of $C_{n}^{2}$ apparently. On the other hand, the cone effect depends on the vertical distribution of the lateral wind speed as well. The cone effect becomes more obvious as the zenith angle increases. In comparison to a near infrared wavelength, the cone effect becomes larger in the case of the visible wavelength. In all cases concerned in this paper, an AO system by using a sodium guide star has almost same phase compensation effectiveness as that by using the astronomical target itself as a beacon.
\end{abstract}

KEY WORDS: Adaptive optics system, Numerical simulation, Laser guide star, Cone effect, Adaptive optics telescope, Phase compensation effectiveness

\section{INTRODUCTION}

It is well known that an astronomical adaptive optics (AO) system can greatly improve the astronomical observation on the Earth. However, knowledge of the aberrated wavefront is the critical input for an AO system and the AO system needs a bright enough beacon (in astronomy this beacon is called as a guide star) within the isoplanatic field for the real-time measurement of the wavefront. ${ }^{1,2}$ This requirement limits the use of $\mathrm{AO}$ for astronomy. Therefore, in 1985, it is suggested to use a laser to generate artificial guide star at arbitrary positions on the sky: ${ }^{3}$ the laser is focused into the atmosphere at small angle from the target object, and backscattering light, i.e. the "laser guide star (LGS)" or "laser beacon", is used by the wavefront sensor to measure the aberrated wavefront. Either Rayleigh or Mie scattering off air molecules at moderate altitudes (up to about $20 \mathrm{~km}$ ) and resonant scattering by sodium (Na) atoms at an altitude of about $90 \mathrm{~km}$ are powerful ways to obtain the LGS. ${ }^{4}$

One of the main two problems introduced by LGS is the consequence of the finite altitude of the LGS. The LGS

\footnotetext{
* Correspondence: Email: hxyan@imech.ac.cn; Phone: (86) 10 62554123; Fax: (86) 1062561284.
} 
emits a spherical wavefront, which does not pass through the same turbulence as a natural star or astronomical target. Nevertheless, the natural star is corrected by using the wavefront mearements of the LGS. The induced error is called the cone effect (or focus anisoplanatism). It induces a systemic erroneous correction and thus limits improvement of the image quality. The cone effect becomes more critical toward shorter wavelength, and for larger telescope apertures.

The cone effect comes from the finite height of the artificial guide star above the telescope. The light rays from the astronomical target, which is at infinite or very great distance, form a parallel bundle and pass through the atmosphere as a cylinder, while the rays from the LGS trace out a cone. The turbulence above the LGS is not sampled at all, and the difference in the propagation paths (i.e. between the cylinder and the cone) leads to an incorrect sampling of the turbulence below the LGS. For the sodium guide stars, which are formed well above the troposphere, the first of these two effects is negligible, and it is thought that the mismatch for the lower turbulence is less severe than for Rayleigh guide stars.

During the recent 30 years, the cone effect for the LGS and solutions to eliminating it in the astronomy with adaptive optics have been widely investigated., ${ }^{2,4}$ However, almost all of these investigations are by means of the analytical theory. For example, in China, Shen investigated an important problem - modal decomposition of the anisoplanatic errors in his doctoral thesis. ${ }^{6}$ Based on the Zernike polynomials, the statistical correlation of random wavefronts between the LGS and the target was studied, and the focus anisoplanatism and the angular anisoplanatism were unified. The mathematical expressions for modal decomposition and the analytic relationships for modal anisoplanatism of planar and spherical wave were presented. Viard and his colleagues studied the four-LGS method for improving the image quality when an AO system is used in an astronomical telescope. ${ }^{7}$ Based on an analytical study, the residual cone effect with four LGSs was investigated and presented. Furthermore, in order to overcome the cone effect Buscher el al. proposed an alternative method to sense the wavefront distortion produced during the beam's return passage of a LGS. ${ }^{8}$ A key advantage of their method is that a parallel beam is used, and therefore the wavefront measurements will not suffer from the cone effect.

Generally, an AO system can be divided into three parts: wavefront detection, wavefront reconstruction and wavefront correction. Moreover, there are three important factors to affect the performances of the AO system. The first of them is the limited spatial bandwidth resulted from the limited numbers of sub-apertures in the wavefront sensor for wavefront detection and actuators in the deformable mirror for wavefront correction. The second is effects of noise and detection error. The third is the limited temporal bandwidth resulted from the limited response speed for wavefront detection, reconstruction and correction. The present authors have been pursuing modeling and numerical simulation of atmospheric propagation of optical waves and an AO system since 1988. ${ }^{9-14}$ In Ref. [9], a comprehensive theoretical model has been developed and coded into a numerical algorithm. A numerical simulation of the atmospheric propagation of an optical wave in the turbulence and a numerical simulation of the operation of an AO system in a static state were combined. The distorted wavefront after propagating through the atmospheric turbulence is detected, reconstructed and corrected by the AO system. In that paper, the direct wavefront gradient control method was used to reconstruct the wavefront phase and the long-exposure Strehl ratio was used to evaluate the performances of the AO system. In Ref. [10], a theoretical model of numerical simulation of an AO system by modal wavefront reconstruction (i.e. by means of the Zernike polynomials expansion) was presented and a corresponding computer program was compiled. A numerical simulation investigation of an AO system by means of the modal wavefront reconstruction was carried out in that paper. The first factor to affect the AO system performances, i.e. the effect of limited spatial bandwidth was investigated in Refs. $[9,10]$. Refs. [11,12] focused on the effects of noise and detection error and on the 
dynamic control process and frequency response characteristics in an AO system, respectively. Thus, a comprehensive modeling and numerical simulation of an AO system were completed. Based on our theoretical model and computer program, the effects of reconstruction matrix and controller coefficients on performances of an AO system and the interaction of the effects of noise and detection error with the dynamic control process in an AO system were investigated by a pure numerical simulation. ${ }^{13,14}$

In this paper, the cone effect in astronomy with an $\mathrm{AO}$ system is investigated by means of a pure numerical simulation of atmospheric propagation of optical wave and an adaptive optics (AO) system including dynamic control process. It should be noted that in all the previous investigations of the cone effect which have utilized the analytical formulae, the dynamic control process in an AO system has not been included. However, in practice an AO system must include the dynamic control process as a necessary portion of the AO system and it is thought that the dynamic control process might have important influence on the cone effect, i.e. the cone effect for an AO system including the dynamic control process might have some differences in comparison to that without the dynamic control process. In light of these, this preliminary investigation of the cone effect by using a pure numerical simulation of an exemplary AO system is carried out. Various conditions in the practical application of wave propagation through the turbulent atmosphere and the AO system with a PI (proportional plus integral) controller are included in an analogous way.

\section{NEMERICAL SIMULATION}

The propagation of an optical wave through the turbulent atmosphere is a key problem in this investigation. As in Ref. [9], the multiple phase screen (PS) model is used to describe the turbulent media. The main idea is as follows. The effect of turbulence on an optical wave is expressed in terms of random change in the refractive index. The propagation path through the turbulent media can be divided into several segments which may have different lengths. It is thought that each segment may deform the phase of the optical wave independently but does not have a significant influence on the amplitude of the wave. The amplitude of the optical wave is changed only in the propagation process of the wave with the deformed phase. It means that changes in amplitude and phase of the optical wave can be treated separately. Then, the contribution of the turbulent media segment to the phase of the optical wave can be "pressed" into a very thin phase screen and added to the initial phase of the wave. The wave with changed phase then propagates to the next phase screen in a media without turbulence. At the position of the next phase screen, the wave with changed amplitude is modified by the new phase screen and so on. This process continues until the wave reaches the target.

Following Dr. Li of the Institute of Optics and Electronics, Chinese Academy of Sciences, ${ }^{15}$ eight atmospheric models, i.e. distribution profiles of $C_{n}^{2}$ vs. altitude, are used in this paper. The well-known Hufnagel-Valley (HV) model and the modified HV models are included in them. Equations relating the altitude $h$ (in meter) to $C_{n}^{2}$ are:

HV0: $C_{n}^{2}=8.16 \times 10^{-54} h^{10} e^{-h / 1000}+3.02 \times 10^{-17} e^{-h / 1500}+1.43 \times 10^{-14} e^{-h / 100}$

Modified HV1: $C_{n}^{2}=8.16 \times 10^{-54} h^{10} e^{-h / 1000}+3.02 \times 10^{-17} e^{-h / 1500}+1.9 \times 10^{-15} e^{-h / 100}$

Modified HV2: $C_{n}^{2}=8.16 \times 10^{-54} h^{10} e^{-h / 1000}+3.02 \times 10^{-17} e^{-h / 1500}+6.4 \times 10^{-15} e^{-h / 100}$

$\mathrm{HV}-21: C_{n}^{2}=5.94 \times 10^{-53}(\omega / 27)^{2} h^{10} e^{-h / 1000}+2.7 \times 10^{-16} e^{-h / 1500}+A e^{-h / 100}$ 


$$
\omega=21 \mathrm{~m} / \mathrm{s}, A=1.7 \times 10^{-14}
$$

HV-25: $C_{n}^{2}=5.94 \times 10^{-53}(\omega / 2)^{2} h^{10} e^{-h / 1000}+2.7 \times 10^{-16} e^{-h / 1500}+A e^{-h / 100}$

$$
\omega=25.7 \mathrm{~m} / \mathrm{s}, A=3.189 \times 10^{-16}
$$

$\mathrm{HFD:} \quad C_{n}^{2}=5.3 \times 10^{-50} h^{10} e^{-h / 610}+4 \times 10^{-15} e^{-h / 300}+1.8 \times 10^{-16} e^{-h / 430}$

$\mathrm{HFN}: C_{n}^{2}=2.3 \times 10^{-52} h^{10} e^{-h / 990}+3.9 \times 10^{-16} e^{-h / 190}+1.2 \times 10^{-16} e^{-h / 330}$

$$
\text { SLC-day: } \quad C_{n}^{2}=\left\{\begin{array}{cc}
0 & 0 m<h<19 m \\
4.008 \times 10^{-13} h^{-1.054} & 19 m<h<230 m \\
1.3 \times 10^{-15} & 230 m<h<850 m \\
6.352 \times 10^{-7} h^{-2.996} & 850 m<h<7000 m \\
6.209 \times 10^{-16} h^{-0.6229} & 7000 m<h<20000 m
\end{array}\right.
$$

A common feature of all these models is that $C_{n}^{2}$ is largest on the ground; as altitude increases, $C_{n}^{2}$ decreases rapidly; there is a region having a weak turbulence around the altitude of $2 \mathrm{~km}$ for most of the models; and there is a region having a stronger turbulence around the altitude of 5-10 km. According to this feature, we select an arrangement of the multiple phase screens. After numerical experiment, 15 phase screens are chosen, and their distribution is as follows: 5 phase screens in 0-500 m, divided equally, i.e. one per $100 \mathrm{~m} ; 3$ phase screens in 500-2000 m, divided equally, i.e. one per $500 \mathrm{~m} ; 5$ phase screens in $2-12 \mathrm{~km}$, divided equally, i.e. one per $2 \mathrm{~km} ; 2$ phase screens in $12-20 \mathrm{~km}$, divided equally, i.e. one per $4 \mathrm{~km}$; and the atmospheric turbulence is omitted in the region above $20 \mathrm{~km}$. This arrangement is based on the following consideration: fully utilizing not too many phase screens to obtain more accurate computational results in view of that the computer hours are proportional to the number of phase screens; utilizing more phase screens in the region having stronger turbulence and rapid variation of $C_{n}^{2}$ vs. altitude; utilizing less and less phase screens as the turbulence getting weaker and the variation of $C_{n}^{2}$ vs. altitude getting slower.

The average $C_{n}^{2}$ at positions of the 15 phase screens and the atmospheric coherence length $r_{0-I R}$ of the whole propagation path for a near infrared (IR) wavelength by using these eight models are summarized in Table 1 . The corresponding figure of the $C_{n}^{2}$ distributions vs. altitude for the eight atmospheric models is shown in Fig. 1.

It is shown from Table 1 and Fig. 1 that the $C_{n}^{2}$ magnitudes and distributions for the eight models are quite different. For $r_{0-I R}$ of the whole propagation path, values for models HV1, HV2, HV25 and HFN are larger, i.e. the atmospheric turbulence for them is weaker. In fact, the distribution of $C_{n}^{2}$ vs. altitude has more important influence on the astronomical observation on the ground which influence is the main interested topic in this paper.

For the distribution of lateral wind speed vs. altitude, a commonly used model of the wind speed is used in this paper. The equation relating the lateral wind speed to the altitude $v_{w}(h)$ is expressed as:

$$
v_{w}(h)=v_{g}+30 \exp \left\{-[(h-9400) / 4800]^{2}\right\} \quad(\mathrm{m} / \mathrm{s}), \text { taking } v_{g}=5 \mathrm{~m} / \mathrm{s} .
$$

The distribution of the wind speed vs. the altitude is shown in Table 1 as well. Its corresponding figure is Fig. 2. 


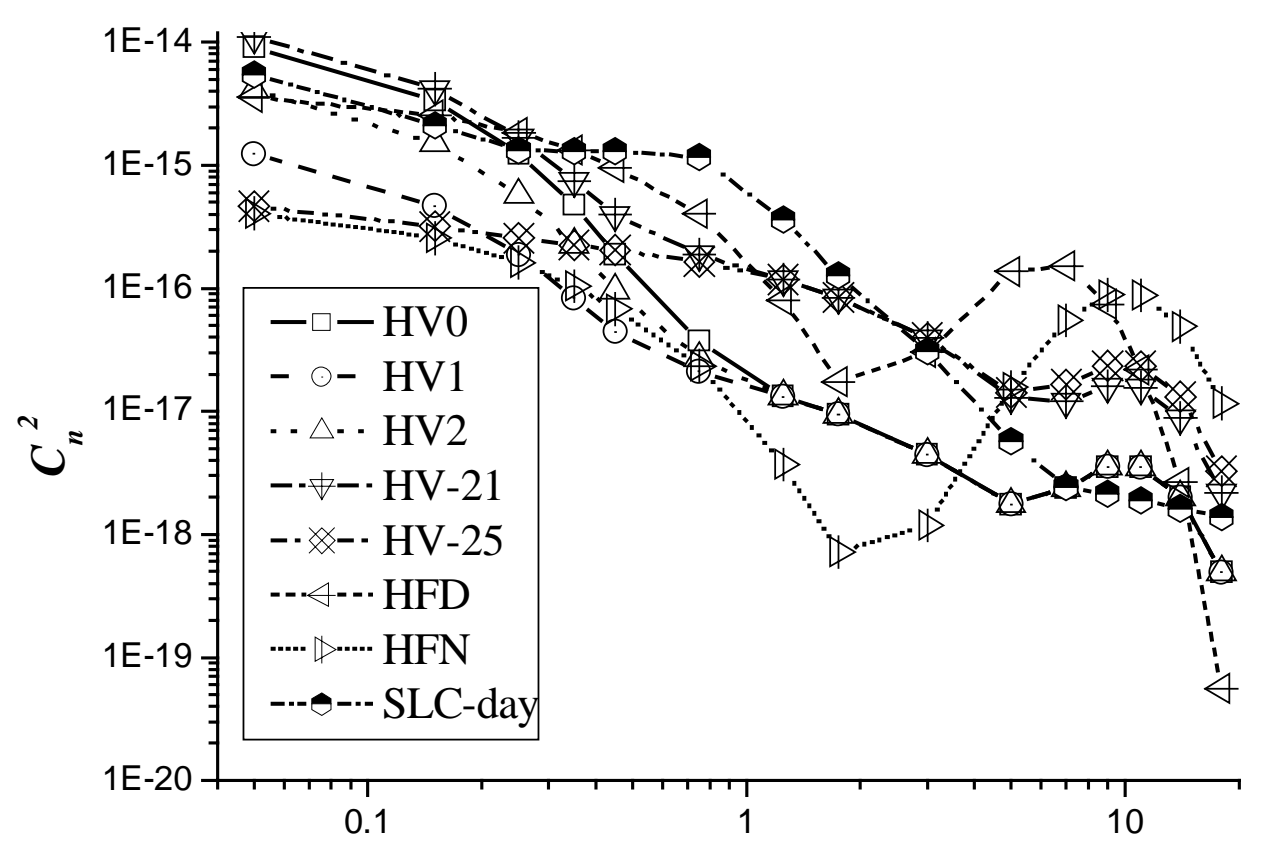

Altitude (km)

Fig. 1. Distributions of $C_{n}^{2}$ vs. altitude $\boldsymbol{h}$ for eight atmospheric turbulence models.

In another aspect, an exemplary AO system is utilized in this paper. The configuration of the deformable mirror (DM) actuators and the Hartmann-Shack (HS) sensor subapetures in this AO system is same as that in Ref. [13]. There are 32 actuators locating on the grids of 20 square subapetures. The diameter of the telescope is assumed to be 0.6 meter. Similar to in Ref. [13], the coupling factor between adjacent actuators is taken as 0.15 ; and the distance between the adjacent actuators is taken as $12 \mathrm{~cm}$. In Ref. [13], we proposed a new approach to produce a modified reconstruction matrix and it was shown by a numerical simulation investigation that this matrix can

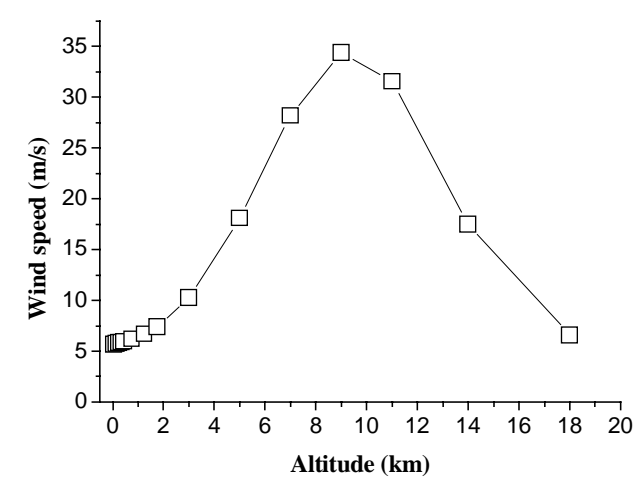

Fig. 2. Distributions of the wind speed $v_{w}$ vs. altitude $h$.

produce better phase compensation effectiveness and much better long time period working stability in an AO system. This modified reconstruction matrix is utilized in this paper. In present preliminary investigation, the dynamic control process of an $\mathrm{AO}$ system is included, but the effects of noise and detection error are not included.

Some other concrete simulation conditions are as follows. An optical wave propagation scene of a geostationary satellite which altitude is about $36 \times 10^{3} \mathrm{~km}$ is chosen in this paper. The altitudes of the Rayleigh guide star are chosen 
as 4,10 , and $20 \mathrm{~km}$; and the altitude of the sodium guide star is chosen as $90 \mathrm{~km}$. The beacon wavelength of the Rayleigh guide star is taken as $532 \mathrm{~nm}$; and the beacon wavelength of the sodium guide star is taken as $589 \mathrm{~nm}$ as in the practice.

Table 1. Average $C_{n}^{2}$ and average $v_{w}$ at positions of 15 phase screens and the atmospheric coherence length $r_{0-I R}$ of the whole propagation path for a near IR wavelength by using eight atmospheric models

\begin{tabular}{|c|c|c|c|c|c|}
\hline \multicolumn{2}{|c|}{ Model } & HV0 & HV1 & HV2 & HV-21 \\
\hline \multicolumn{2}{|c|}{$r_{0-I R}(\mathrm{~cm})$} & 20.075 & 56.957 & 31.380 & 15.836 \\
\hline \multicolumn{2}{|c|}{$\begin{array}{c}\text { Altitude of PS } \\
h(\mathrm{~km})\end{array}$} & \multicolumn{4}{|c|}{ Average $C_{n}^{2}\left(\mathrm{~m}^{-2 / 3}\right)$} \\
\hline & 0.05 & 0.9072 E-14 & $0.1231 \mathrm{E}-14$ & $0.4076 \mathrm{E}-14$ & $0.1101 \mathrm{E}-13$ \\
\hline & 0.15 & 0.3354 E-14 & $0.4693 \mathrm{E}-15$ & $0.1516 \mathrm{E}-14$ & 0.4199 E-14 \\
\hline & 0.25 & 0.1249 E-14 & 0.1882 E-15 & 0.5733 E-15 & 0.1684 E-14 \\
\hline & 0.35 & $0.4741 \mathrm{E}-15$ & 0.8374 E-16 & $0.2254 \mathrm{E}-15$ & 0.7491 E-15 \\
\hline & 0.45 & $0.1880 \mathrm{E}-15$ & 0.4438 E-16 & $0.9650 \mathrm{E}-16$ & $0.3970 \mathrm{E}-15$ \\
\hline & 0.75 & $0.3760 \mathrm{E}-16$ & 0.2095 E-16 & $0.2700 \mathrm{E}-16$ & 0.1874 E-15 \\
\hline & 1.25 & 0.1332 E-16 & $0.1320 \mathrm{E}-16$ & $0.1324 \mathrm{E}-16$ & 0.1180 E-15 \\
\hline & 1.75 & 0.9449 E-17 & 0.9449 E-17 & 0.9449 E-17 & 0.8447 E-16 \\
\hline & 3.00 & 0.4439 E-17 & 0.4439 E-17 & $0.4439 \mathrm{E}-17$ & $0.3950 \mathrm{E}-16$ \\
\hline & 5.00 & 0.1748 E-17 & 0.1748 E-17 & 0.1748 E-17 & 0.1296 E-16 \\
\hline & 7.00 & 0.2400 E-17 & 0.2400 E-17 & $0.2400 \mathrm{E}-17$ & 0.1196 E-16 \\
\hline & 9.00 & $0.3528 \mathrm{E}-17$ & $0.3528 \mathrm{E}-17$ & $0.3528 \mathrm{E}-17$ & 0.1590 E-16 \\
\hline & 1.00 & $0.3512 \mathrm{E}-17$ & $0.3512 \mathrm{E}-17$ & $0.3512 \mathrm{E}-17$ & $0.1556 \mathrm{E}-16$ \\
\hline & 4.00 & $0.2001 \mathrm{E}-17$ & $0.2001 \mathrm{E}-17$ & $0.2001 \mathrm{E}-17$ & $0.8828 \mathrm{E}-17$ \\
\hline & 8.00 & $0.4932 \mathrm{E}-18$ & 0.4932 E-18 & 0.4932 E-18 & 0.2173 E-17 \\
\hline \multicolumn{2}{|c|}{ Model } & HV-25 & HFD & HFN & SLC-day \\
\hline \multicolumn{2}{|c|}{$R_{0-I R}(\mathrm{~cm})$} & 33.842 & 16.369 & 28.203 & 16.547 \\
\hline$h(\mathrm{~km})$ & $v_{w}(h)(\mathrm{m} / \mathrm{s})$ & \multicolumn{4}{|c|}{ Average $C_{n}^{2}\left(\mathrm{~m}^{-2 / 3}\right)$} \\
\hline 0.05 & 5.675 & $0.4629 \mathrm{E}-15$ & 0.3562 E-14 & 0.4068 E-15 & 0.5456 E-14 \\
\hline 0.15 & 5.732 & $0.3185 \mathrm{E}-15$ & $0.2565 \mathrm{E}-14$ & $0.2556 \mathrm{E}-15$ & 0.2127 E-14 \\
\hline 0.25 & 5.793 & 0.2559 E-15 & 0.1847 E-14 & $0.1623 \mathrm{E}-15$ & 0.1330 E-14 \\
\hline 0.35 & 5.858 & $0.2239 \mathrm{E}-15$ & $0.1331 \mathrm{E}-14$ & 0.1042 E-15 & 0.1300 E-14 \\
\hline 0.45 & 5.928 & $0.2038 \mathrm{E}-15$ & $0.9601 \mathrm{E}-15$ & $0.6775 \mathrm{E}-16$ & 0.1300 E-14 \\
\hline 0.75 & 6.172 & 0.1650 E-15 & 0.4011 E-15 & $0.2349 \mathrm{E}-16$ & 0.1160 E-14 \\
\hline 1.25 & 6.686 & 0.1179 E-15 & 0.7997 E-16 & 0.3700 E-17 & 0.3632 E-15 \\
\hline 1.75 & 7.375 & 0.8447 E-16 & $0.1730 \mathrm{E}-16$ & $0.7202 \mathrm{E}-18$ & 0.1273 E-15 \\
\hline 3.00 & 10.26 & 0.3959 E-16 & $0.3021 \mathrm{E}-16$ & 0.1188 E-17 & 0.3073 E-16 \\
\hline 5.00 & 18.07 & $0.1425 \mathrm{E}-16$ & 0.1366 E-15 & 0.1574 E-16 & 0.5704 E-17 \\
\hline 7.00 & 28.19 & 0.1655 E-16 & $0.1513 \mathrm{E}-15$ & 0.5494 E-16 & 0.2411 E-17 \\
\hline 9.00 & 34.37 & $0.2346 \mathrm{E}-16$ & 0.7408 E-16 & 0.8869 E-16 & $0.2142 \mathrm{E}-17$ \\
\hline 11.00 & 31.55 & $0.2322 \mathrm{E}-16$ & 0.2178 E-16 & 0.8808 E-16 & 0.1889 E-17 \\
\hline 14.00 & 17.50 & $0.1321 \mathrm{E}-16$ & 0.2640 E-17 & 0.4906 E-16 & $0.1629 \mathrm{E}-17$ \\
\hline 18.00 & 6.597 & $0.3253 \mathrm{E}-17$ & 0.5586 E-19 & 0.1165 E-16 & 0.1391 E-17 \\
\hline
\end{tabular}

In a practical AO system, a controller for controlling the dynamic process must be included and a dynamic iteration computation of thousands time steps must be carried out. ${ }^{12,13}$ A difference equation

$$
U_{n}=a_{1} U_{n-1}+a_{2} U_{n-2}+a_{3} U_{n-3}+\cdots+b_{1} E_{n}+b_{2} E_{n-1}+b_{3} E_{n-2}+\cdots
$$


connects the corrected wavefront at the $n$th time step $U_{n}$ with the corrected wavefronts at the foregoing time steps $U_{n-l}$, $U_{n-2}, \ldots$ and the residual wavefronts at the present time step and the foregoing time steps $E_{n}, E_{n-1}, E_{n-2}, \ldots$ A different controller shows different performances in terms of the difference of this connection, i.e. in terms of the different coefficients in this equation. A simpler PI (proportional plus integral) controller is utilized and the controller coefficients $a_{l}=0.94, b_{1}=0.50$, others $=0$ are chosen in this paper. The frame rate in the AO system is chosen as $1500 \mathrm{~Hz}$ and 3000 dynamic iterations are utilized to obtain the statistically average simulation results in this paper.

\section{RESULT AND DISCUSSION}

We propose to use a parameter $S R$ as an evaluation parameter to express the phase compensation effectiveness of an AO system. The $S R$ is defined as:

$$
S R=S T R C C \text { (closed loop) / STRCC (open loop). }
$$

wherein STRCC (closed loop) is the Strehl ratio STRCC for the AO system with a closed loop (i.e. having the phase compensation of the AO system), and STRCC (open loop) is the STRCC for the AO system with an open loop (i.e. without an AO system). The STRCC is defined as a ratio of the optical energy within a circle around the centroid (optical center) with a radius of the first dark ring in the Airy pattern after propagations through the turbulent medium to that through a vacuum. ${ }^{9}$ The long-exposure STRCC is utilized in this paper. In the numerical simulation of an AO system including dynamic control process, a large number of patterns at target corresponding to different time steps of dynamic iteration are accumulated to obtain a long-exposure pattern. In calculating the long-exposure STRCC, the centroid and the Strehl ratio are determined from the accumulated long-exposure pattern at target.

\section{(1) In the case of a near infrared wavelength}

Choosing the zenith angle as 0 degree, computational results of the numerical simulation for eight atmospheric turbulence models are summarized in Table 2. As mentioned above, the dynamic control process of an AO system is included in the simulation, and the $S R$ is chosen as the evaluation parameter of the phase compensation effectiveness of an $\mathrm{AO}$ system. Its corresponding figure is Fig. 3.

Table 2. Dynamic iteration results $S R$ vs. altitude of the beacon in the case of a near infrared wavelength and zenith angle of 0 degree

\begin{tabular}{|c|c|c|c|c|c|c|c|c|c|}
\hline $\begin{array}{c}\text { Altitude } \\
(\mathrm{km})\end{array}$ & $\begin{array}{c}\text { Number } \\
\text { of PS }\end{array}$ & HV0 & HV1 & HV2 & HV-21 & HV-25 & HFD & HFN & SLC-day \\
\hline \multicolumn{2}{|c|}{$r_{\text {-IR }}(\mathrm{cm})$} & 20.075 & 56.957 & 31.380 & 15.836 & 33.842 & 16.369 & 28.203 & 16.547 \\
\hline 4 & 9 & 2.41 & 1.17 & 1.62 & 2.93 & 1.32 & 2.25 & 1.29 & 3.01 \\
\hline 10 & 12 & 2.42 & 1.17 & 1.63 & 3.00 & 1.35 & 2.33 & 1.29 & 3.16 \\
\hline 20 & 15 & 2.43 & 1.17 & 1.63 & 3.04 & 1.37 & 2.46 & 1.33 & 3.20 \\
\hline 90 & 15 & 2.43 & 1.18 & 1.63 & 3.08 & 1.40 & 2.58 & 1.43 & 3.21 \\
\hline 36000 & 15 & 2.43 & 1.18 & 1.63 & 3.07 & 1.41 & 2.59 & 1.44 & 3.21 \\
\hline
\end{tabular}

It is surprisingly found from Table 2 and Fig. 3 that the effect of altitudes of the beacon (LGS or the astronomical target itself) on the AO phase compensation effectiveness by using the commonly-available vertical profiles of the refractive index structure constant $C_{n}^{2}$ in the atmosphere is relatively weak, and the cone effect for some profiles is even negligible. For example, the cone effects for model HV0, HV1 and HV2 is almost negligible, i.e. the curves of $S R$ 
vs. beacon altitude for these models are almost flat. (See Fig. 3)

It is found that the cone effect seems not have obvious relationship with the turbulence strength. The atmospheric coherence lengths $r_{0}$ for models HV0, HV1 and HV2 are quite different (see Table 1 or 2), but the cone effect for these models is similarly negligible, although $S R$ (the phase compensation effectiveness) has obvious difference for them. However, the cone effect depends on the distribution profiles of $C_{n}^{2}$ obviously. When $C_{n}^{2}$ distribution of an atmospheric turbulence model is that $C_{n}^{2}$ is relatively large at lower altitude, especially $C_{n}^{2}$ is very large near the ground, and $C_{n}^{2}$ decreases rapidly as altitude increases, the cone effect for this model is weaker. This is the case for models HV0, HV1 and HV2. On the contrary, if $C_{n}^{2}$ is relatively small at

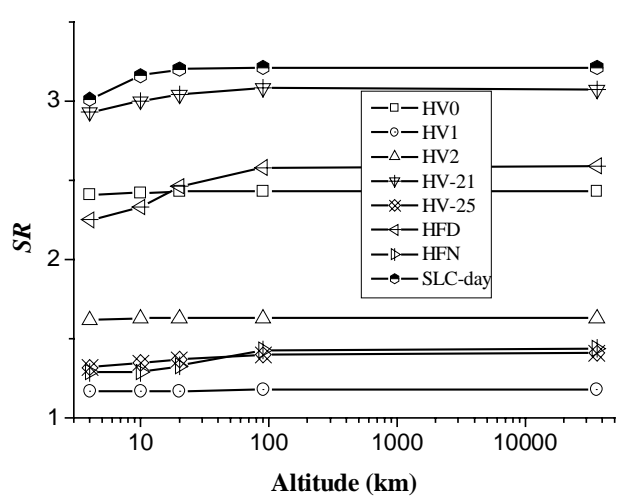

Fig. 3. $S R$ vs. beacon altitude for a near IR wavelength and zenith angle of 0 degree

lower altitude, and especially $C_{n}^{2}$ decreases slowly as altitude increases, the cone effect for this model is more obvious. For example, this is the case of model SLC-day. The reason for these can be understood as follows. As mentioned above, there are two sources to produce the cone effect: the first is that the turbulence above the LGS is not sampled at all; the second is the difference in propagation paths of the astronomical target and the beacon (i.e. between a cylinder and a cone). Because the atmospheric turbulence in the region of higher altitudes is relatively weak, it can be imagined that the influence of the first source has less importance. As far as the second source is concerned, the difference between a cylinder and a cone is smaller at lower altitudes and this difference gets larger and larger as the altitude increases. Thus, in the case that the atmospheric turbulence at higher altitudes is not very weak, the cone effect should be more obvious.

On the other hand, the cone effect depends on the vertical distribution of the lateral wind speed as well. As mentioned above, the numerical simulation results presented in this paper include the effect of dynamic control process in an AO system. For a static target such as a geostationary satellite, the effect of dynamic control process mainly comes from the lateral wind in the atmosphere. It can be seen from Table 1 and Fig. 2 that the lateral wind speed in the atmosphere has a peak around $10 \mathrm{~km}$ altitude. Thus, in the case that the atmospheric turbulence at higher altitudes is not very weak, the cone effect could be more obvious as well.

(2) In the case of different zenith angles

Table 3. Dynamic iteration results $S R$ vs. altitude of the beacon in the case of a near infrared wavelength and zenith angle of 30 degree

\begin{tabular}{|c|c|c|c|c|c|c|c|c|c|}
\hline $\begin{array}{c}\text { Distance } \\
(\mathrm{km})\end{array}$ & $\begin{array}{c}\text { Number } \\
\text { of PS }\end{array}$ & HV0 & HV1 & HV2 & HV-21 & HV-25 & HFD & HFN & SLC-day \\
\hline \multicolumn{2}{|c|}{$R_{\text {O-IR }}(\mathrm{cm})$} & 18.415 & 52.247 & 28.785 & 14.527 & 31.044 & 15.015 & 25.871 & 15.179 \\
\hline 4 & 9 & 2.65 & 1.22 & 1.77 & 3.16 & 1.39 & 2.40 & 1.36 & 3.28 \\
\hline 10 & 12 & 2.67 & 1.22 & 1.78 & 3.27 & 1.44 & 2.51 & 1.37 & 3.52 \\
\hline 20 & 15 & 2.67 & 1.23 & 1.78 & 3.32 & 1.47 & 2.67 & 1.40 & 3.58 \\
\hline 103.68 & 15 & 2.68 & 1.23 & 1.79 & 3.37 & 1.51 & 2.85 & 1.54 & 3.60 \\
\hline 36737 & 15 & 2.68 & 1.23 & 1.79 & 3.37 & 1.52 & 2.86 & 1.55 & 3.60 \\
\hline
\end{tabular}


Choosing the zenith angles as 30,45 and 60 degree, computational results of the numerical simulation for eight atmospheric turbulence models are summarized in Table 3-5, respectively. Their corresponding figures are Fig. 4-6.

It can be seen from Tables 3-5 that as the zenith angle increases, $r_{0-I R}$ becomes smaller, i.e. the atmospheric turbulence strength becomes stronger, and $S R$, i.e. phase compensation effectiveness becomes larger. Further, it can be seen from Tables 3-5 and Figs. 4-6 that the cone effect becomes more obvious as the zenith angle increases.

Table 4. Dynamic iteration results $S R$ vs. altitude of the beacon in the case of

a near infrared wavelength and zenith angle of 45 degree

\begin{tabular}{|c|c|c|c|c|c|c|c|c|c|}
\hline $\begin{array}{c}\text { Distance } \\
(\mathrm{km})\end{array}$ & $\begin{array}{c}\text { Number } \\
\text { of PS }\end{array}$ & HV0 & HV1 & HV2 & HV-21 & HV-25 & HFD & HFN & SLC-day \\
\hline \multicolumn{2}{|c|}{$R_{\text {-IR }}(\mathrm{cm})$} & 16.306 & 46.263 & 25.488 & 12.863 & 27.488 & 13.296 & 22.908 & 13.440 \\
\hline 4 & 9 & 2.94 & 1.25 & 1.91 & 3.42 & 1.45 & 2.71 & 1.37 & 3.58 \\
\hline 10 & 12 & 2.96 & 1.25 & 1.92 & 3.55 & 1.51 & 2.81 & 1.37 & 3.96 \\
\hline 20 & 15 & 2.98 & 1.26 & 1.93 & 3.63 & 1.54 & 2.99 & 1.41 & 4.06 \\
\hline 126.41 & 15 & 2.99 & 1.26 & 1.94 & 3.70 & 1.61 & 3.28 & 1.58 & 4.12 \\
\hline 37632 & 15 & 2.98 & 1.27 & 1.94 & 3.70 & 1.61 & 3.28 & 1.58 & 4.11 \\
\hline
\end{tabular}

Table 5. Dynamic iteration results $S R$ vs. altitude of the beacon in the case of

a near infrared wavelength and zenith angle of 60 degree

\begin{tabular}{|c|c|c|c|c|c|c|c|c|c|}
\hline $\begin{array}{c}\text { Distance } \\
(\mathrm{km})\end{array}$ & $\begin{array}{c}\text { Number } \\
\text { of PS }\end{array}$ & HV0 & HV1 & HV2 & HV-21 & HV-25 & HFD & HFN & SLC-day \\
\hline \multicolumn{2}{|c|}{$R_{0-I R}(\mathrm{~cm})$} & 13.244 & 37.578 & 20.703 & 10.448 & 22.327 & 10.799 & 18.607 & 10.917 \\
\hline 4 & 9 & 3.77 & 1.36 & 2.28 & 4.11 & 1.51 & 2.59 & 1.52 & 3.52 \\
\hline 10 & 12 & 3.84 & 1.37 & 2.31 & 4.41 & 1.59 & 2.78 & 1.53 & 4.36 \\
\hline 20 & 15 & 3.87 & 1.38 & 2.32 & 4.57 & 1.65 & 2.96 & 1.55 & 4.64 \\
\hline 176.40 & 15 & 3.88 & 1.39 & 2.34 & 4.65 & 1.74 & 3.28 & 1.72 & 4.75 \\
\hline 38836 & 15 & 3.88 & 1.39 & 2.33 & 4.63 & 1.74 & 3.26 & 1.69 & 4.74 \\
\hline
\end{tabular}

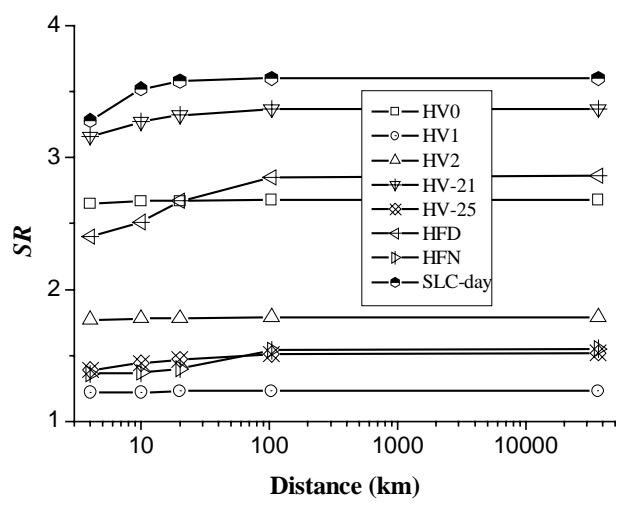

Fig. 4. SR vs. beacon distance for a near IR wavelength and zenith angle of 30 degree

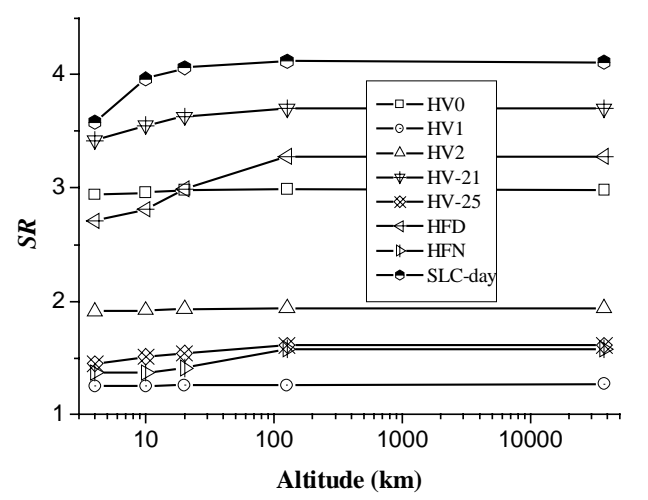

Fig. 5. SR vs. beacon distance for a near IR wavelength and zenith angle of 45 degree 


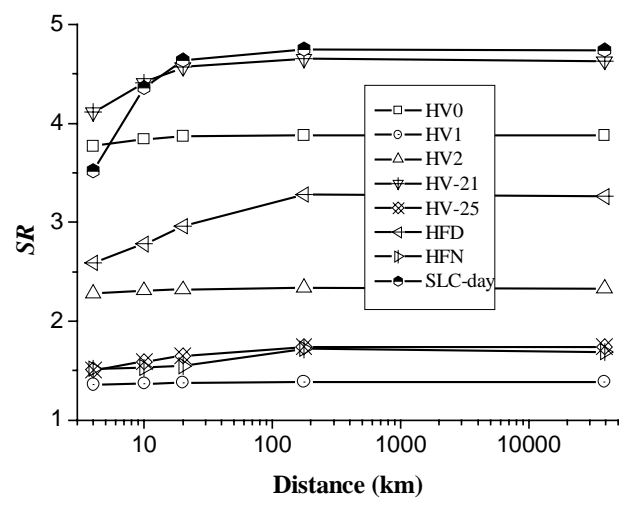

Fig. 6. $S R$ vs. beacon distance for a near IR wavelength and zenith angle of 60 degree

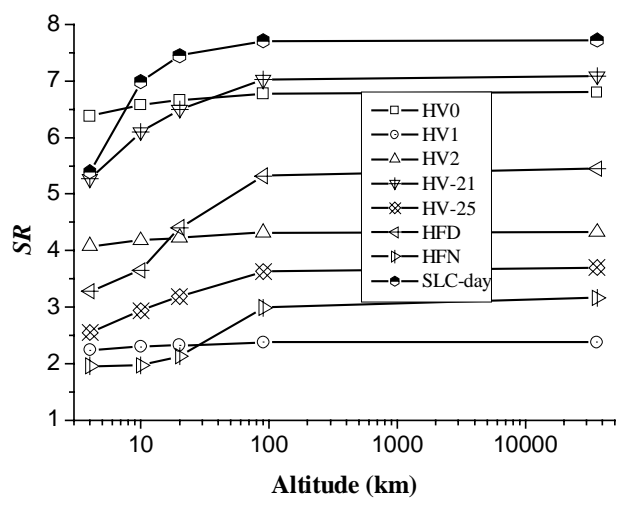

Fig. 7. $S R$ vs. beacon altitude for the visible wavelength and zenith angle of 0 degree

\section{(3) In the case of the visible wavelength}

Choosing the zenith angles as 0 degree and the light wavelength as $550 \mathrm{~nm}$, computational results of the numerical simulation for eight atmospheric models are summarized in Table 6. Its corresponding figure is Fig. 7.

It can be seen from Table 6 that in comparison to a near infrared wavelength, $r_{0-V}$ becomes smaller, i.e. the atmospheric turbulence strength becomes stronger and $S R$, i.e. phase compensation effectiveness of an AO system becomes apparently larger in the case of the visible wavelength. Further, it can be seen from Table 6 and Fig. 7 that the cone effect becomes more obvious as well.

Table 6. Dynamic iteration results $S R$ vs. altitude of the beacon in the case of

The visible wavelength and zenith angle of 0 degree

\begin{tabular}{|c|c|c|c|c|c|c|c|c|c|}
\hline $\begin{array}{c}\text { Altitude } \\
(\mathrm{km})\end{array}$ & $\begin{array}{c}\text { Number } \\
\text { of PS }\end{array}$ & HV0 & HV1 & HV2 & HV-21 & HV-25 & HFD & HFN & SLC-day \\
\hline \multicolumn{2}{|c|}{$r_{\text {O-V }}(\mathrm{cm})$} & 7.053 & 20.01 & 11.02 & 5.564 & 11.89 & 5.751 & 9.909 & 5.814 \\
\hline 4 & 9 & 6.38 & 2.24 & 4.08 & 5.27 & 2.55 & 3.28 & 1.96 & 5.39 \\
\hline 10 & 12 & 6.58 & 2.30 & 4.18 & 6.10 & 2.94 & 3.65 & 1.97 & 6.99 \\
\hline 20 & 15 & 6.66 & 2.32 & 4.23 & 6.50 & 3.19 & 4.41 & 2.13 & 7.45 \\
\hline 90 & 15 & 6.78 & 2.38 & 4.32 & 7.02 & 3.63 & 5.32 & 2.99 & 7.71 \\
\hline 36000 & 15 & 6.80 & 2.38 & 4.33 & 7.09 & 3.70 & 5.45 & 3.16 & 7.72 \\
\hline
\end{tabular}

(4) Further indication of effect of $C_{n}^{2}$ distribution vs. altitude

In order to understand influence of $C_{n}^{2}$ distribution vs. altitude on the cone effect better, two imaginary $C_{n}^{2}$ distributions are produced for models HV0 and SLC-day. In them it is assumed that $C_{n}^{2}$ is a constant at any altitude along the light path, i.e. $C_{n}^{2}$ distribution vs. altitude is even. At the same time, $r_{0}$ of the whole light path is maintained to be same as those in their original models. For the zenith angle of 0 degree and a near IR wavelength, computational results for these two imaginary (even) distributions and their normal ones are summarized in Table 7. Its corresponding figure is Fig. 8.

It can be seen from Table 7 and Fig. 8 that the cone effect becomes much larger for the even $C_{n}^{2}$ distributions. 
This is consistent with our explanation to the cone effect described above.

Table 7. $S R$ vs. altitude of the beacon for a near IR wavelength and zenith angle of 0 degree, showing influence of $C_{n}^{2}$ distribution vs. altitude Wherein "Nor." denotes the normal $C_{n}^{2}$ distribution, and "Even" denotes an even (an imaginary) $C_{n}^{2}$ distribution

\begin{tabular}{|c|c|c|c|c|l|}
\hline Altitude & Number & \multicolumn{2}{|c|}{ HV0 } & \multicolumn{2}{c|}{ SLC-day } \\
\cline { 3 - 6 }$(\mathrm{km})$ & of PS & Nor. & Even & Nor. & Even \\
\hline 4 & 9 & 2.41 & 1.54 & 3.01 & 1.69 \\
\hline 10 & 12 & 2.42 & 1.60 & 3.16 & 1.78 \\
\hline 20 & 15 & 2.43 & 1.69 & 3.20 & 1.91 \\
\hline 90 & 15 & 2.43 & 1.92 & 3.21 & 2.25 \\
\hline 36000 & 15 & 2.43 & 1.94 & 3.21 & 2.27 \\
\hline
\end{tabular}

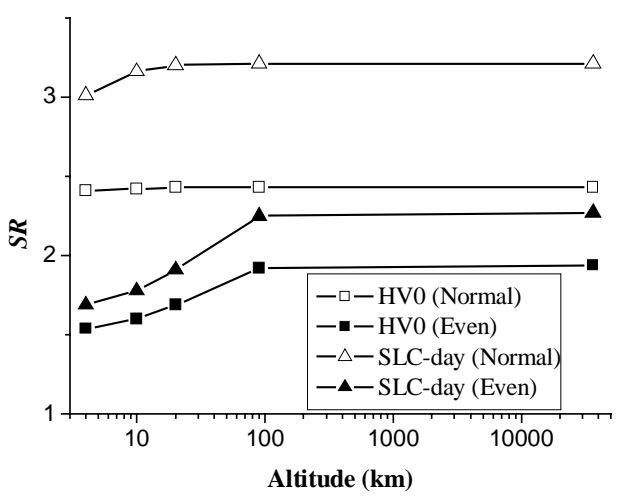

Fig. 8. $S R$ vs. beacon altitude for a near IR wavelength and zenith angle of 0 degree, showing influence of $C_{n}^{2}$ distribution

\section{CONCLUSION}

By using an exemplary AO system, including the dynamic control process in a numerical simulation of the AO system, and proposing $S R$ as the evaluation parameter, the cone effect in astronomy with an AO system is investigated by means of a pure numerical simulation in this paper for the first time within our knowledge, although it is a preliminary numerical simulation investigation. Some conclusions can be deduced from this study: (1) It is surprisingly found that the effect of altitudes of the beacon (LGS or the astronomical target itself) on the AO phase compensation effectiveness by using the commonly-available vertical profiles of the refractive index structure constant $C_{n}^{2}$ and the lateral wind speed in the atmosphere is relatively weak, and the cone effect for some profiles is even negligible. (2) It is found that the cone effect seems not have obvious relationship with the turbulence strength, however, it depends on the vertical distribution profile of $C_{n}^{2}$ apparently. On the other hand, the cone effect depends on the vertical distribution of the lateral wind speed as well. (3) The cone effect becomes more obvious as the zenith angle increases. (4) In comparison to a near infrared wavelength, the cone effect becomes larger in the case of the visible wavelength. (5) In all cases concerned in this paper, an $\mathrm{AO}$ system by using the sodium guide star has almost same phase compensation effectiveness as that by using the astronomical target itself as a beacon. Although this study is carried out by utilizing an exemplary AO system and by using some concrete conditions and it is preliminary, we believe that the conclusions deduced from it are commonly applicable.

\section{REFERENCE}

1. M. C. Roggemann and B. M. Welsh, Imaging Through Turbulence, CRC Press, Boca Raton, Florida, 1996.

2. R. K. Tyson, ed., Adaptive Optics Engineering Handbook, Dekker, New York, 2000.

3. R. Foy and A. Labeyrie, "Feasibility of adaptive optics telescope with laser probe," Astron. Astrophys. 152, pp. L29-L31, 1985.

4. N. Ageorges and J. C. Dainty, eds., Laser Guide Star Adaptive Optics for Astronomy, Kluwer Academic, Dordrecht, 
The Netherlands, 2000.

5. F. Roddier, ed., Adaptive Optics in Astronomy, Cambridge University press, 1999.

6. Feng Shen, "Technologies of laser guide star and low level light wavefront sensing for adaptive optics," Doctoral Thesis, Institute of Optics and Electronics, Chinese Academy of Sciences, 2002. (in Chinese)

7. E. Viard, M. Le Louarn and N. Hubin, "Adaptive optics with four laser guide stars: correction of the cone effect in large telescope," Appl. Opt. 41 (1), pp. 11-20, 2002.

8. D. F. Buscher, G. D. Love and R. M. Myers, "Laser beacon wave-front sensing without focal anisoplanatism," Opt. Lett. 27 (3), pp. 149-151, 2002

9. Hai-Xing Yan, Shu-Shan Li, De-Liang Zhang, and She Chen, "Numerical simulation of an adaptive optics system with laser propagation in the atmosphere," Appl. Opt. 39 (18), pp.3023-3031, 2000.

10. Hai-Xing Yan, She Chen, De-Liang Zhang, and Shu-Shan Li, "Numerical simulation of an adaptive optics system by means of modal wavefront reconstruction," Acta Optica Sinica 18 (1), pp. 103-108, 1998. (in Chinese)

11. Hai-Xing Yan, She Chen, and Shu-Shan Li, "Numerical simulation investigations of the effects of noise and detection error in an adaptive optics system," Proc. SPIE Vol. 4494, pp.144-155, 2002.

12. Hai-Xing Yan, Shu-Shan Li, and She Chen, "Numerical simulation investigations of the dynamic control process and frequency response characteristics in an adaptive optics system," Proc. SPIE Vol. 4494, pp.156-166, 2002.

13. Hai-Xing Yan, Shu-Shan Li, and She Chen, "Effects of reconstruction and controller coefficients on performances of an adaptive optics system investigated by a numerical simulation," Proc. SPIE Vol. 4926, pp. 81-91, 2002.

14. She Chen, Hai-Xing Yan, and Shu-Shan Li, "Effects of noise and detection error in a dynamic adaptive optics system," Proc. SPIE Vol. 4926, pp. 107-118, 2002.

15. Xinyang Li, Internal Report, Institute of Optics and Electronics, Chinese Academy of Sciences, Chengdu, 2002. (in Chinese) 